\title{
Advancing Our CSWB Dialogue Into Year Two
}

\author{
Norman E. Taylor*
}

I begin by welcoming our growing body of readers and contributors into our second year of publication. As we launch this first issue of our Volume 2, we are pleased to introduce another diverse series of articles that reflects and reinforces the stated mission of our Journal of CSWB.

The Journal was recently presented an opportunity to not only express that mission aloud, but also to link it directly to some important work underway across the CSWB movement in Canada. In partnership with others, our parent organization CSKA organized a January event entitled Measuring Collaborative Risk-driven Intervention, billed as the first "interactive national dialogue on research, evaluation and analysis of the Hub/Situation Table and related CSWB models". Over 60 academics, evaluators, policy makers, and practitioners came together in Toronto to take stock of the nation-wide experience to date surrounding multi-sector approaches to CSWB. We hope to publish a more complete record of those proceedings in our next issue.

As Editor-in-Chief of the Journal, I was honoured to accept the invitation to serve as the dinner speaker for this event. For those interested, the full text of my address can be accessed at www.cskacanada.ca. And, as a Journal "first", we have included a Podcast version in connection with this editorial which can be accessed at https://journalcswb.ca/ index.php/cswb/issue/view/4. We hope to do more with this type of multi-media feature in the future. For now, I'd like to highlight just this brief passage:

"Our Journal is committed to advancing the social science ... and thus informing and shaping the public policy ... that will be necessary to conceiving, launching, proving concept, proliferating, funding and sustaining progressive and socially innovative practices ... policies and practices that are born of research, evidence and scientific knowledge where it can be found or created, supported by data where it is or where it can be mined and shared, and at all times, anchored in a renewed collaboration among all parts of the public service system to meet the Community Safety and Well-Being ambitions and outcomes that all our citizens deserve."

I believe this first issue of 2017 once again gives proof to our continuing commitment to those ambitions, as we feature here a number of articles with the potential to advance dialogue, to influence public policy, and to shorten the distance between research, practice, and alignment across our public sector and community-based human services.

We are for the first time activating an editorial section that has thus far been silent, "CSWB Data Analytics \& Technologies". Both of that section's articles in this issue, from Kutnowski and Capotosto, will introduce readers new to this discussion with a necessary appeal to the ethical questions arising from a data-driven world of human services. We hope other contributors will soon weigh into this discussion with us, and that future issues will also bring more evidence in support of, or in challenge to, the ways in which analytics and technologies can do no harm, on the one hand, while improving social outcomes, on the other.

Advancing on data-driven and evidence-based practices in CSWB will continue to challenge the policy and budgetary considerations of public sector organizations in every jurisdiction. We know that traditional investments and entrenched practices do not yield easily to still-forming social innovations. Doyle \& McFee offer a well-developed argument for alternative social financing strategies, with a view to opening new channels that may prove more agile in the short term, and much more accountable in the long term.

The Muise \& Mackey article takes us through two very promising social innovation projects that, while outlining many worthy and promising observations on the models themselves, also highlight these same dimensions of systemic change, tracing the dual challenge of introducing and sustaining new approaches to meeting some very intractable and too-often tragic conditions.

Reflecting the spirit of dialogue that we hope to achieve through the Journal, Bell's letter to the Editor offers a direct response and brings additional insight to the Huey commentary on harm-focused policing from last November's Vol. 1(3) of the Journal.

And finally, this current issue features only one piece of original research, but it is one that CSWB practitioners, policymakers, and community stakeholders have long awaited. Returning to the event described above, the participants on hand in January exhibited a strong appetite for harder evidence in the form of quantitative and economically supported argument amidst a growing body of positive qualitative indicators that continue to endorse the collaborative interventions of the Hub/Situation Table model. The Sawatsky, Ruddell \& Jones paper is the first original research piece to weigh into this space, with a cost-of-crime-based examination of just some of the evident economic impacts resulting 
locally from Canada's original Prince Albert Hub model. As any good researchers should do, these authors take pains to outline the limitations of this first such study. But, they also introduce us to some very promising numbers, derived from a well-disciplined thesis study, which will undoubtedly encourage other researchers to follow their lead and continue to build our understanding of the full potential of CSWB collaboration.

We trust you will derive full value from all of these readings, and as always, on behalf of our whole editorial team,
I look forward to your feedback on this commentary or on any of the articles in this first issue of 2017.

CONFLICT OF INTEREST DISCLOSURE

The author has no known conflicts of interest.

AUTHOR AFFILIATIONS

*Editor-in-Chief, Journal of Community Safety and Well Being. 